Supporting Information

\title{
Facet-Dependent in situ Growth of Nanoparticles in Epitaxial Thin Films: The Role of Interfacial Energy
}

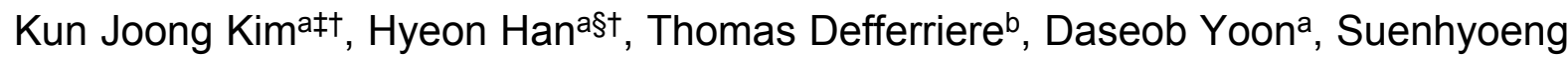
$\mathrm{Na}^{\mathrm{a}}$, Sun Jae Kimª, Amir Masoud Dayaghia, Junwoo Sona, Tae-Sik Oh ${ }^{\mathrm{c}}$, Hyun Myung Jang $^{\mathrm{a} \# *}$ and Gyeong Man Choi ${ }^{\mathrm{a} d *}$

aDepartment of Materials Science \& Engineering / Fuel Cell Research Center, Pohang University of Science and Technology (POSTECH), Pohang 37673, Republic of Korea ${ }^{b}$ Department of Materials Science and Engineering, Massachusetts Institute of Technology, Cambridge, MA, 02139, USA

'Department of Chemical Engineering, Auburn University, Auburn, AL 36849, USA d1FCell Inc., Pohang 37673, Republic of Korea

fPresent address: Department of Materials Science and Engineering, Massachusetts Institute of Technology, Cambridge, MA, 02139, USA

\$Present address: Max Planck Institute of Microstructure Physics, Weinberg 2, 06120 Halle (Saale), Germany

\#Present address: Research Institute of Advanced Materials, Seoul National University, Seoul 08826, Republic of Korea

†These authors contributed equally to this work.

*Corresponding authors: hmjang@postech.ac.kr, gmchoi@postech.ac.kr 

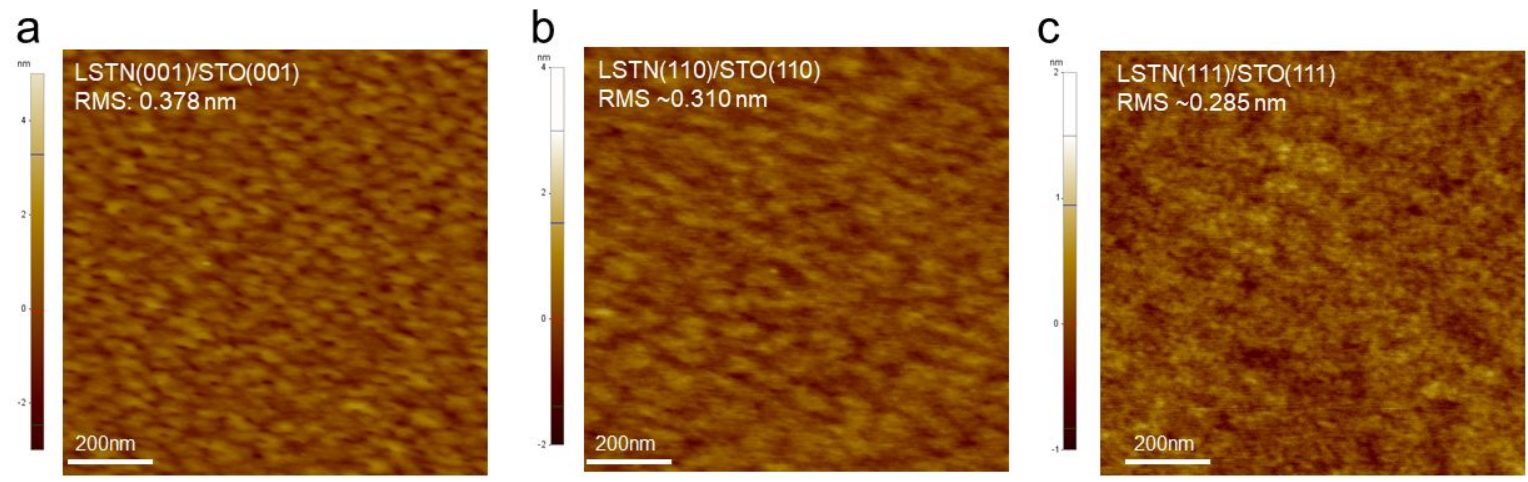

Figure S1. AFM images of as-deposited samples. (a) (001)-, (b) (110)-, and (c) (111)oriented films. 

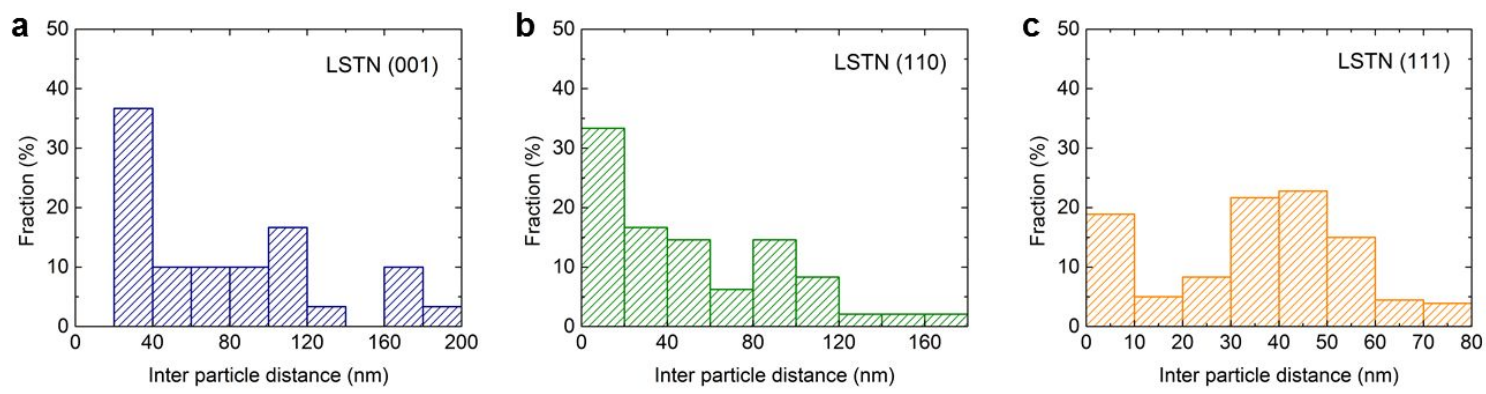

Figure S2. Distribution of inter-particle distance (IPD) for (a) LSTN (001), (b) LSTN (110), and (c) LSTN (111) films. The IPD was calculated for an area of $1 \mu \mathrm{m}^{2}$ in the SEM images using ImageJ software. 
Table S1. Comparison of the experimental $d$-spacing values for LSTN film and STO substrate obtained from the SAED patterns in Figure 2 with the theoretical d-spacing for five planes. Herein, the theoretical values were calculated from the bulk lattice parameters of the $\mathrm{SrTiO}_{3}(\mathrm{STO})$ perovskite $(a=b=c=3.905 \AA)$. The difference in dspacing values for STO substrate and bulk consistently shows $\sim 5.7 \%$; this discrepancy does stem from instrumental errors such as accuracy of camera length, wavelength, and etc.

\begin{tabular}{ccccc} 
& LSTN (001) & \multicolumn{2}{c}{ STO (001) } & \\
\hline \hline Plane & $\begin{array}{c}\text { Experimental } \\
\text { value }\end{array}$ & $\begin{array}{c}\text { Experimental } \\
\text { value }\end{array}$ & $\begin{array}{c}\text { Theoretical } \\
\text { value }\end{array}$ & $\begin{array}{c}\text { Degree of } \\
\text { matching }\end{array}$ \\
$(011)$ & 0.2928 & 0.2931 & 0.2761 & $106.1 \%$ \\
$(002)$ & 0.2060 & 0.2059 & 0.1952 & $105.5 \%$ \\
$(0 \overline{1} 1)$ & 0.2923 & 0.2926 & 0.2761 & $106.0 \%$ \\
$(001)$ & 0.4121 & 0.4119 & 0.3905 & $105.5 \%$ \\
$(0 \overline{1} 3)$ & 0.1304 & 0.1303 & 0.1234 & $105.5 \%$
\end{tabular}



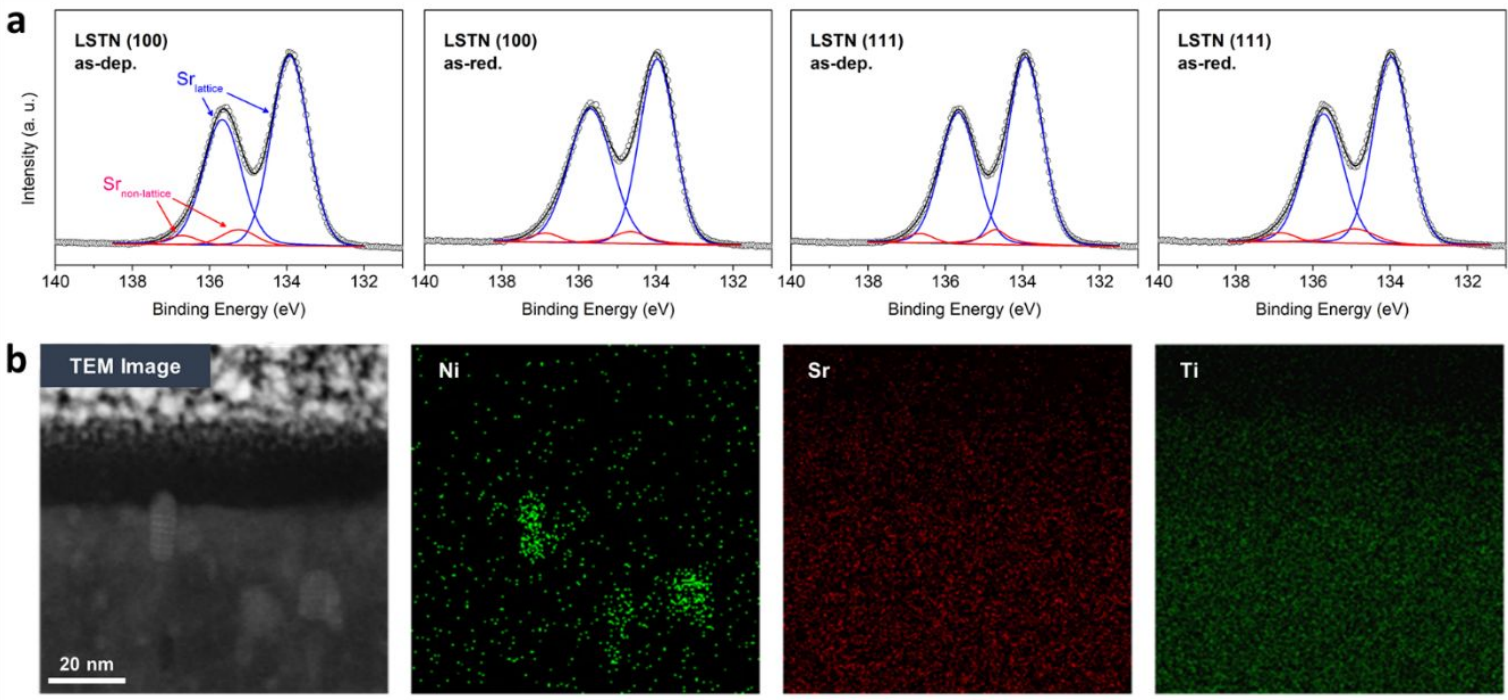

Figure S3. The possibility of existing other composition such as $\mathrm{SrO}$ on the surface. (a) XPS spectra of $\mathrm{Sr} 3 \mathrm{~d}$. The blue doublets correspond to the bulk coordinated $\mathrm{Sr}_{\text {lattice }}$ and the red doublets are attributed to SrO phase formation on the surface coordinated $\mathrm{Sr}_{\text {non-lattice }}$ (Refs. 21-25 in the main manuscript). From left to right, the spectra of asdeposited (001)-oriented, as-reduced (001)-oriented, as-deposited (111)-oriented, and as-reduced (111)-oriented thin films are shown. The proportion of $\mathrm{Sr}_{\text {non-lattice }}$ peak area is $5.8 \%, 6.1 \%, 6.2 \%$ and $6.5 \%$, respectively. (b) A cross-sectional STEM image of LSTN (001) surface and corresponding EDS maps of $\mathrm{Ni}, \mathrm{Sr}$, and $\mathrm{Ti}$; it shows that the $\mathrm{Ni}$ atoms are solely clustered together. 

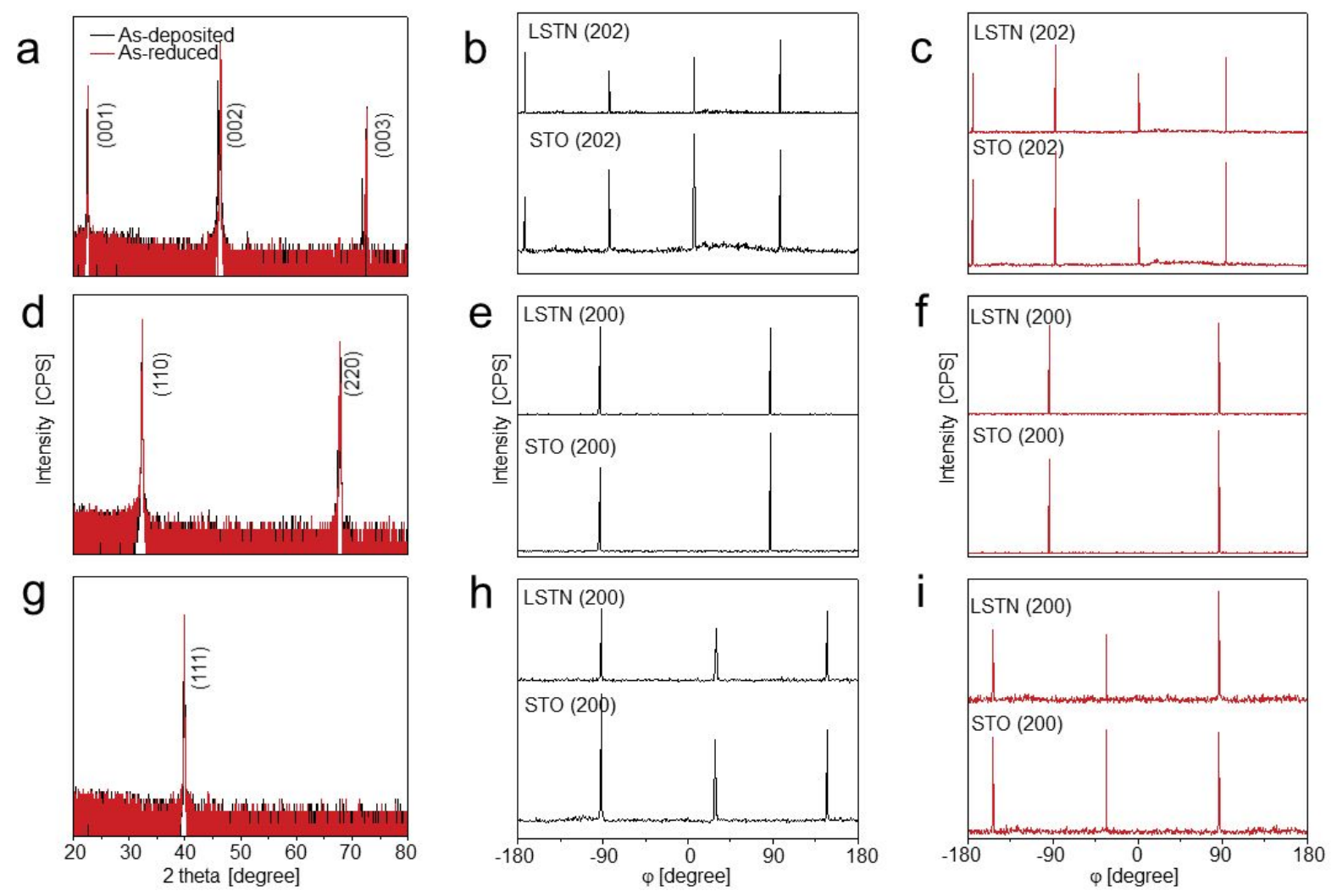

Figure S4. Wide $\theta-2 \theta$ scan of the LSTN films deposited on (a) STO(001), (c) (110), and (e) (111) substrate. The observed $\{001\}$ (a), $\{110\}$ (d), and $\{111\}$ (g) XRD peaks indicate that the LSTN films are highly oriented. No impurity phases are observed within the detection sensitivity of XRD.

$(b, c)$ In-plane $\varphi$-scan spectra of the LSTN (202) and STO (202) planes for the (001)oriented LSTN film grown on STO. b: as-deposited, c: as-reduced.

$(e, f)$ In-plane $\varphi$-scan spectra of the LSTN (200) and STO (200) planes for the (110)oriented LSTN film grown on STO. b: as-deposited, c: as-reduced.

$(\mathrm{h}, \mathrm{i})$ In-plane $\varphi$-Scan spectra of the LSTN (200) and STO (200) planes for the (111)oriented LSTN film grown on STO. b: as-deposited, c: as-reduced.

The two, three, and four fold symmetry with uniformly separated peaks can be found in (001)-, (110)-, and (111)-oriented films, confirming the existence of epitaxial relationship between LSTN thin films and STO substrates. 


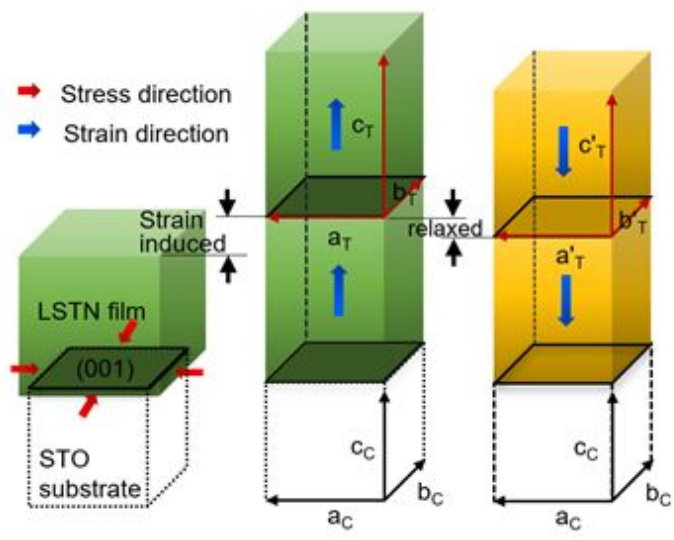

(001)-oriented tetragonal

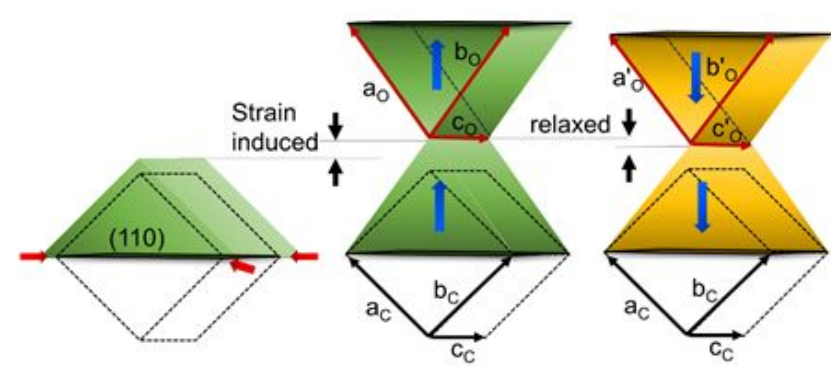

(110)-oriented Orthorhombic

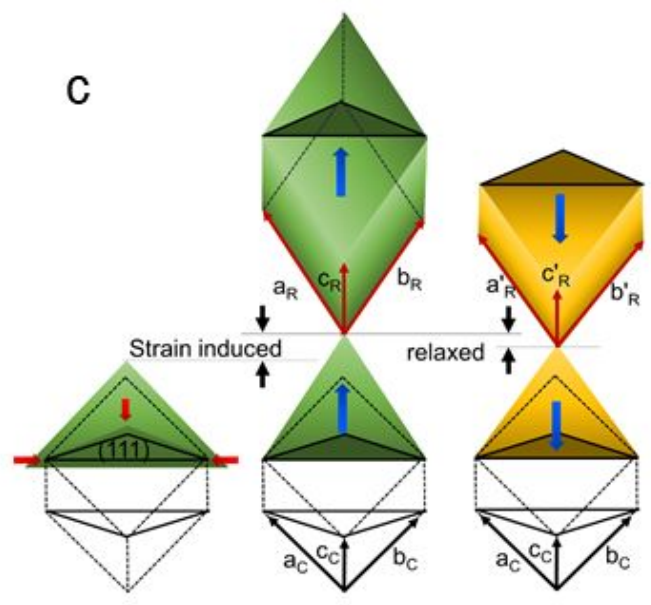

(111)-oriented Rhombohedral

Figure S5. Structure and stress of variously-oriented LSTN films. (a) Tetragonal structure of (001)-oriented film (b) Orthorhombic (or monoclinic) structure of (110)-oriented film (c) Rhombohedral structure of (111)-oriented film, $a_{c}, b_{c}, c_{c}$ : Lattice Parameters $L P$ for cubic, $a_{T}$, $\mathrm{b}_{\mathrm{T}}, \mathrm{c}_{\mathrm{T}}$ : $L P$ for strain-induced tetragonal, $\mathrm{a}_{\mathrm{T}}^{\prime}, \mathrm{b}_{\mathrm{T}} \mathrm{c}_{\mathrm{T}}^{\prime}: L P$ for strain-relaxed tetragonal. $\mathrm{a}_{\mathrm{o}}, \mathrm{b}_{\mathrm{o}}, \mathrm{c}_{\mathrm{o}}$ : $L P$ for strain-induced orthorhombic, $a_{0}^{\prime}, b^{\prime}{ }_{0} c_{0}^{\prime}$ : $L P$ for strain-relaxed orthorhombic. $a_{R}, b_{R}, c_{R}$ : $L P$ for strain-induced rhombohedral, $a_{R}^{\prime}, b_{R}^{\prime} c_{R}^{\prime}: L P$ for strain-relaxed rhombohedral. 

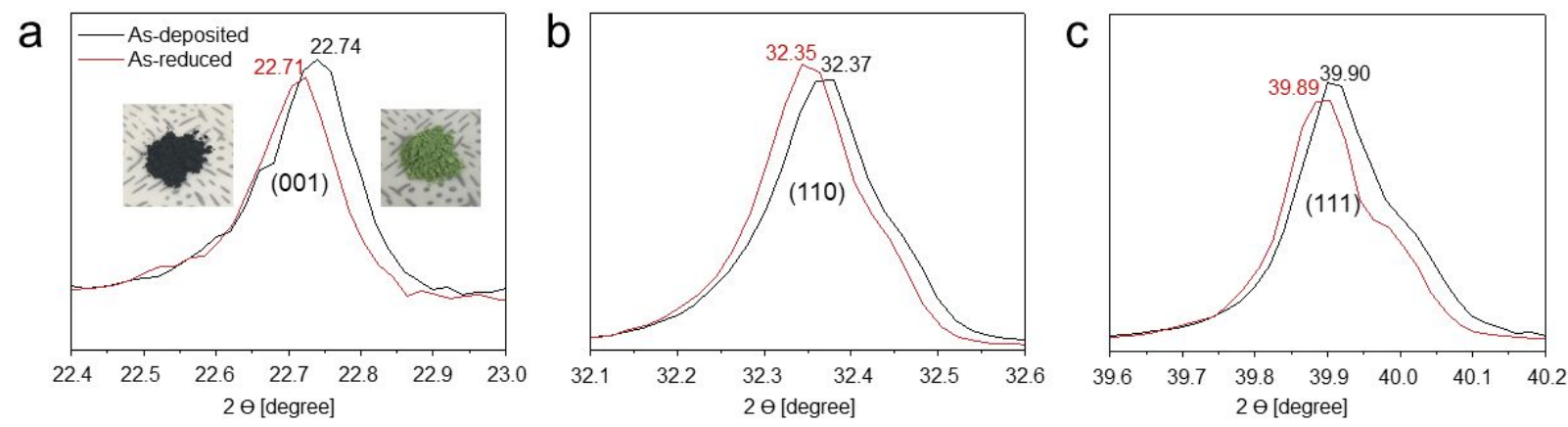

Bulk LSTN

\begin{tabular}{lcc} 
& As-prepared & As-reduced \\
\hline \hline Last thermal history & $1500{ }^{\circ} \mathrm{C} / 5 \mathrm{~h} /$ air & $900{ }^{\circ} \mathrm{C} / 12 \mathrm{~h} / \mathrm{dry} \mathrm{H}_{2}$ \\
Lattice parameter $[\AA \hat{}]$ & $3.9087 \pm 0.0003$ & $3.9104 \pm 0.0002$ \\
Cell volume $\left[\AA^{3}\right]$ & $59.7171 \pm 0.0116$ & $59.7943 \pm 0.0083$ \\
\hline
\end{tabular}

Figure S6. $\theta-2 \theta$ XRD scan of the bulk for (001), (110) and (111) reflection, and difference in lattice parameters and cell volume of as-prepared and as-reduced samples was shown. 
Table S2. A Quantification of the XPS spectra for (001)- and (111)-oriented film. RSF represents the corresponding relative sensitivity factor. The $\mathrm{Ni}^{0}$ spectra for $3 p$ level was excluded. The composition of as-deposited film is $\mathrm{La}_{0.23} \mathrm{Sr}_{0.73} \mathrm{Ti}_{0.93} \mathrm{Ni}_{0.07} \mathrm{O}_{2.95}$ for (001)-oriented film and $\mathrm{La}_{0.22} \mathrm{Sr}_{0.71} \mathrm{Ti}_{0.92} \mathrm{Ni}_{0.08} \mathrm{O}_{2.94}$ for (111)-oriented film. After the reduction, the composition of (001)- and (111)-oriented films are changed to $\mathrm{La}_{0.24} \mathrm{Sr}_{0.75} \mathrm{Ti}_{0.97} \mathrm{Ni}_{0.03} \mathrm{O}_{3.02}$ and $\mathrm{La}_{0.24} \mathrm{Sr}_{0.74} \mathrm{Ti}_{0.96} \mathrm{Ni}_{0.04} \mathrm{O}_{2.97}$, respectively.

\begin{tabular}{|c|c|c|c|c|c|}
\hline & & \multicolumn{2}{|c|}{ LSTN (001) } & \multicolumn{2}{|c|}{ LSTN (111) } \\
\hline & & as-dep. & as-red. & as-dep. & as-red. \\
\hline & La & 0.23 & 0.24 & 0.22 & 0.24 \\
\hline & $\mathrm{Sr}$ & 0.73 & 0.75 & 0.71 & 0.74 \\
\hline \multirow[t]{4}{*}{ Composition } & $\mathrm{Ti}$ & 0.93 & 0.97 & 0.92 & 0.96 \\
\hline & $\mathrm{Ni}$ & 0.07 & 0.03 & 0.08 & 0.04 \\
\hline & $\mathrm{O}$ & 2.95 & 3.02 & 2.94 & 2.97 \\
\hline & Peak area (a.u.) & 34,775 & 31,811 & 35,925 & 29,845 \\
\hline \multirow[t]{3}{*}{ La 4d } & RSF & 2.475 & 2.475 & 2.475 & 2.475 \\
\hline & Peak area/RSF & 14,050 & 12,853 & 14,515 & 12,059 \\
\hline & Peak area (a.u.) & 82,215 & 75,673 & 85,880 & 69,522 \\
\hline \multirow[t]{3}{*}{$\mathrm{Sr} 3 d$} & RSF & 1.843 & 1.843 & 1.843 & 1.843 \\
\hline & Peak area/RSF & 44,610 & 41,060 & 46,598 & 37,722 \\
\hline & Peak area (a.u.) & 113,038 & 105,625 & 121,153 & 97,817 \\
\hline \multirow[t]{3}{*}{ Ti $2 p$} & RSF & 2.001 & 2.001 & 2.001 & 2.001 \\
\hline & Peak area/RSF & 56,491 & 52,786 & 60,546 & 48,884 \\
\hline & Peak area (a.u.) & 2,670 & 1,111 & 3,112 & 1,312 \\
\hline \multirow[t]{3}{*}{ Ni 3p } & RSF & 0.598 & 0.598 & 0.598 & 0.598 \\
\hline & Peak area/RSF & 4,464 & 1,858 & 5,204 & 2,194 \\
\hline & Peak area (a.u.) & 140,297 & 128,847 & 150,889 & 118,495 \\
\hline \multirow[t]{2}{*}{$01 \mathrm{~s}$} & RSF & 0.780 & 0.780 & 0.780 & 0.780 \\
\hline & Peak area/RSF & 179,868 & 165,189 & 193,448 & 151,917 \\
\hline Total & Peak area/RSF & 299,482 & 273,746 & 320,311 & 252,776 \\
\hline
\end{tabular}



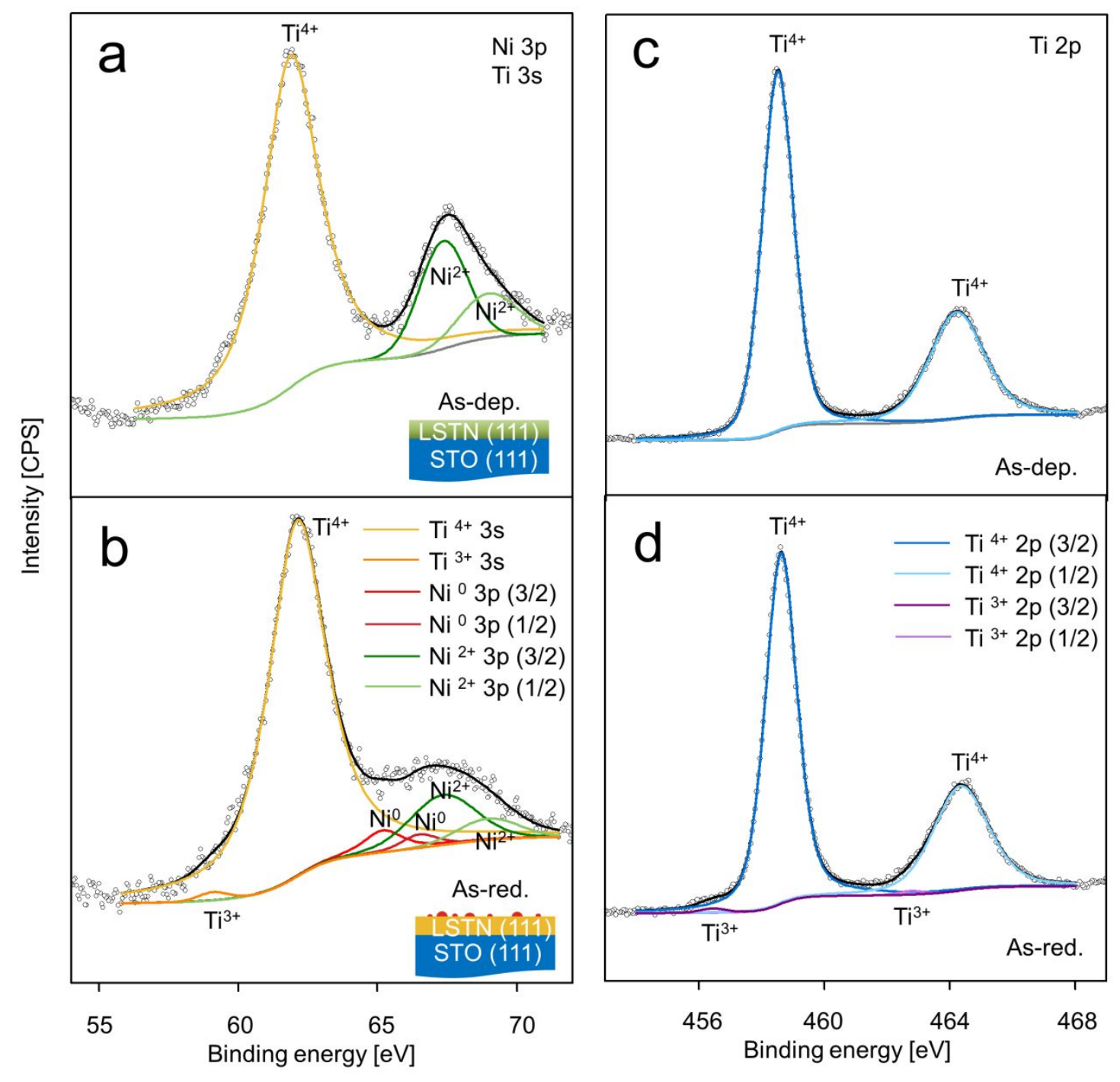

Figure S7. X-ray photoelectron spectroscopy (XPS) for (111)-oriented film. Ni 3p, Ti 3 s of sample before (a) and after (b) exsolution. Ti $2 p$ of samples before (c) and after (d) exsolution. $24 \%$ of surface $\mathrm{Ni}^{0}$ was exsolved from total $\mathrm{Ni}$ amount $\left(\mathrm{Ni}^{0}+\mathrm{Ni}^{2+}\right)$ and $\mathrm{Ti}^{3+} /\left(\mathrm{Ti}^{3+}+\mathrm{Ti}^{4+}\right) \sim 0.8 \%$. 
Table S3. Out-of-plane (OOP) tensile strain (\%) of (001)-, (110)-, (111)-oriented LSTN films. The d-spacing values of as-deposited films and as-prepared bulk were obtained from the XRD data (Figure S4) using Bragg's law. Herein, OOP tensile strain is defined as $f=\frac{d_{\text {film }}-d_{\text {bulk }}}{d_{\text {bulk }}}$

\begin{tabular}{cccc}
\hline \hline & \multicolumn{2}{c}{ d-spacing $[\AA]$} & $\begin{array}{c}\text { oop tensile } \\
\text { strain [\%] }\end{array}$ \\
\cline { 2 - 3 } Plane & bulk & film & \\
$(001)$ & 3.908 & 3.938 & 0.76 \\
$(110)$ & 2.764 & 2.771 & 0.27 \\
$(111)$ & 2.257 & 2.260 & 0.17 \\
\hline \hline
\end{tabular}




\section{Supplementary Note: Calculation of elastic constant E}

In a cubic material, the $\mathrm{E}$ can be determined along any orientation.

The following shows the procedure based on the classical elastic theory ${ }^{1,2}$ for calculating the compliance and direction cosines along the different crystallographic directions. Young's modulus for an arbitrary crystallographic direction is given by

$$
\frac{1}{E_{i j k}}=S_{11}-2\left(S_{11}-S_{12}-\frac{1}{2} S_{44}\right)\left(l_{i 1}^{2} l_{j 2}^{2}+l_{j 2}^{2} l_{k 3}^{2}+l_{i 1}^{2} l_{k 3}^{2}\right)
$$

Where $S$ and / represent three independent elastic compliances and direction cosines respectively of the direction [ijk].

For simplicity, we use elastic compliances of undoped-SrTiO${ }_{3}^{3}$.

$\mathrm{S}_{11}=3.77 \mathrm{TPa}^{-1}, \mathrm{~S}_{12}=-0.93 \mathrm{TPa}^{-1}, \mathrm{~S}_{44}$ is $8.23 \mathrm{TPa}^{-1}$

Direction cosines

and

direction cosine, $\left(l_{i 1}^{2} l_{j 2}^{2}+l_{j 2}^{2} l_{k 3}^{2}+l_{i 1}^{2} l_{k 3}^{2}\right)$

\begin{tabular}{|cccc|}
\hline & $l_{i 1}$ & $l_{j 2}$ & $l_{k 3}$ \\
{$[100]$} & 1 & 0 & 0 \\
{$[110]$} & $1 / \sqrt{2}$ & $1 / \sqrt{2}$ & 0 \\
{$[111]$} & $1 / \sqrt{3}$ & $1 / \sqrt{3}$ & $1 / \sqrt{3}$ \\
\hline
\end{tabular}

is 0 for [100], $1 / 4$ for [110] and $1 / 3$ for [111],

Therefore,

$$
\begin{array}{ll}
\frac{1}{E_{100}}=3.77 & E_{100}=265.25 \quad[\mathrm{GPa}] \\
\frac{1}{E_{111}}=3.77-2\{(3.77+0.93)-8.23 / 2\}\left(\frac{1}{3}\right)=3.38 & E_{110}=287.56 \quad[\mathrm{GPa}] \\
\frac{1}{E_{110}}=3.77-2\{(3.77+0.93)-8.23 / 2\}\left(\frac{1}{4}\right)=3.4775 & E_{111}=295.86 \quad[\mathrm{GPa}]
\end{array}
$$

The largest strain occurs in (001)-oriented film. 


\section{References used in the Supporting Information}

1 W. A. Brantley, Calculated elastic constants for stress problems associated with semiconductor devices, J. Appl. Phys., 1973, 44, 534-535.

2 R. O. Bell and G. Rupprecht, Elastic constants of strontium titanate, Phys. Rev., 1963, 129, 90-94.

3. Handbook of Optics, Volume 5, Atmospheric optics, modulators, fiber optics, Xray and neutron optics. Bass, Michael. New York: McGraw-Hill Professional. 2010. 